Math. Model. Nat. Phenom.

Vol. 9, No. 4, 2014, pp. 131-152

DOI: $10.1051 / \mathrm{mmnp} / 20149409$

\title{
A Review of Optimal Chemotherapy Protocols: From MTD towards Metronomic Therapy
}

\author{
U. Ledzewicz ${ }^{1}$, H. Schättler ${ }^{2}$ \\ ${ }^{1}$ Dept. of Mathematics and Statistics, Southern Illinois University Edwardsville, \\ Edwardsville, Illinois, 62026-1653, USA \\ 2 Dept. of Electrical and Systems Engr., Washington University, St. Louis, Missouri, 63130-4899, USA
}

\begin{abstract}
We review mathematical results about the qualitative structure of chemotherapy protocols that were obtained with the methods of optimal control. As increasingly more complex features are incorporated into the mathematical model-progressing from models for homogeneous, chemotherapeutically sensitive tumor cell populations to models for heterogeneous agglomerations of subpopulations of various sensitivities to models that include tumor immunesystem interactions - the structures of optimal controls change from bang-bang solutions (which correspond to maximum dose rate chemotherapy with restperiods) to solutions that favor singular controls (representing reduced dose rates). Medically, this corresponds to a transition from standard MTD (maximum tolerated dose) type protocols to chemo-switch strategies towards metronomic dosing.
\end{abstract}

Keywords and phrases: optimal control, cancer chemotherapy, homogeneous and heterogeneous tumor populations, tumor immune system interactions

Mathematics Subject Classification: 92C50, 49K15

\section{Introduction: On the Question of Drug Dosing in Chemotherapy}

The question of drug dosing in cancer chemotherapy - albeit the most fundamental and important oneto this day eludes clear quantitative answers. Modern oncology increasingly takes the point of view of a tumor as an agglomeration of subpopulations of cancerous cells of varying therapeutic sensitivities embedded into its microenvironment. This consists of the tumor vasculature, tumor immune system interactions, and several other structures (e.g., fibroblasts and the extra cellular matrix, ...), all embedded into healthy tissue. If specific aspects are isolated, clear and simple answers about the structure of optimal chemotherapy protocols can be given. For example, if a homogenous tumor population of chemotherapeutically sensitive cells is assumed and other aspects of the tumor microenvironment are ignored, then it would seem clear that an optimal treatment schedule should give as much chemotherapy as tolerable. This led to the concept of maximum tolerated doses (MTD), a commonly used paradigm in medical practice. But as other features of the tumor microenvironment are taken into account, the picture becomes blurry and the question of drug dosing eludes clear answers.

${ }^{*}$ Corresponding author. E-mail: uledzew@siue.edu 
It is here where mathematical modeling can help by giving qualitative insights into the structure of optimal therapy protocols by answering questions of the following type: When is an MTD strategy optimal? In which situations can a protracted administration of agents at significantly lower dose rates (the same total dose, but spread in time) achieve better effects? The history of mathematical modeling of cancer treatments is long going back to the 1970s [6] and 1980s [44,46]. In these early, typically noncompartmental models the tumor was considered a homogeneous unit consisting of chemotherapeutically sensitive cells and optimal treatment protocols simply are constant at maximum dose rates. This also makes sense medically: when the disease has progressed into an advanced stage (and these typically were the initial conditions considered), it is imperative to kill as many of the cancer cells as possible and this has to done right now. But drugs are rarely selective in their activation mechanisms and most chemotherapy severely damages other proliferating cells that are essential for survival, such as, for example, the bone marrow. This necessitates the introduction of rest periods for the patient to recover. Such thinking has led to the still commonly favored chemotherapy schedules of maximum dose therapy sessions with restperiods. Early mathematical models all support such a scheme as optimal (e.g., see [6,23,24, 28], [44]-[49] and many more). However, these models implicitly assume a homogenous population of chemotherapeutically sensitive cells. This need not be the case. Cancer cells are genetically unstable and when this gets coupled with high proliferation rates, another main characteristic of many malignant cancers, this leads to significantly higher mutation rates than in healthy cells $[9,10]$. In addition, growing tumors often exhibit considerable evolutionary ability to enhance cell survival in an environment that is becoming hostile [8]. This leads to increased drug resistance of some strains of the cells. As a result, many tumors are populations that consist of cells of widely varying sensitivities towards a particular chemotherapeutic agent. For example, the Norton-Simon hypothesis postulates that tumors typically consist of faster growing cells that are sensitive to chemotherapy and slower growing populations of cells that exhibit lower sensitivities or are resistant to the chemotherapeutic agent. There may even exist (possibly tiny) subpopulations of cells that are not sensitive to the treatment from the beginning (ab initio, intrinsic resistance). Given such a scenario, over time, as the drugs kill sensitive tumor cells, the proportions of the resistant subpopulation of cancer cells increases and may become dominant. In such a scenario, an MTD-style therapy will cause more harm to the healthy cells than deliver beneficial effects $[25,26,50]$.

The question how chemotherapeutic agents should be scheduled to optimize their effects therefore is a difficult one. While MTD chemotherapy has been the staple of medical practice - and it certainly seems the best approach in many cases - it is not without it pitfalls and in medical trials to this day other options are explored. The MTD approach focusses on cancer cells, but ignores other aspects of the tumor microenvironment such as its vasculature or tumor-immune system interactions. The question simply is whether the format of optimal treatment protocols changes if these additional aspects are taken into account. For example, it has been shown in medical trials that low dose chemotherapy can have both antiangiogenic and immune-stimulatory effects (e.g., see $[5,19,35]$ ). Hypothetically, if most sensitive cells have been eliminated, but there exists a small residuum of chemotherapeutically resistant cells, MTD chemotherapy at a later stage may negatively effect beneficial actions of the immune system while its effect on the tumor is insignificant. For, without this additional therapy boost actions of the immune system might have been able to control the small residual resistant tumor (immune surveillance), but then get eliminated by the extra chemotherapy session allowing the small resistant tumor to break free and become malignant. On the other hand, low-dose chemotherapy of the same agent might stimulate the immune system which then may be able to control this tiny surviving residuum preventing its growth into a fully developed tumor. Metronomic chemotherapy, "the chronic administration of chemotherapy at relatively low, minimally toxic doses on a frequent schedule of administration, at close regular intervals, with no prolonged drug-free breaks" $[1,15]$ is one of several alternatives that are pursued in medical research to take advantage of such effects (e.g., see $[2,3]$ ). By administering drugs in a continuous low-dose way, it is hoped to avoid the limiting toxic side effects of high dose therapy and possibly the overall effect of therapy might be improved because of the greatly extended time horizon over which drugs can be given when compared with repeated MTD doses: "concentration $\times$ time" matters [16,52]. Similar ideas also 
underlie so-called "chemo-switch" regimens [36] which follow initial MTD sessions with administrations of agents at much reduced dose rates later on. But the ultimate question-how to optimize the antitumor, anti-angiogenic and pro-immune effects of chemotherapy by modulating dose and administration schedule - so far has no general satisfactory answer when the true system (patient) is considered. This is just one more of the many challenges with the "multifaceted disease" cancer [7].

In this paper, we review some results that were obtained for mathematical models of cancer chemotherapy with the methods of optimal control. As increasingly more complex mathematical models are considered that incorporate various aspects of the tumor microenvironment, optimal solutions no longer are necessarily of the MTD-type, but instead favor adminstration of agents at lower dose rates.

\section{Homogeneous Tumor Populations: MTD Protocols}

We start with a mathematical model for cancer chemotherapy for a homogeneous tumor population of chemotherapeutically sensitive cells. The model, originally formulated and analyzed by Swierniak in [47], is cell-cycle specific and, in addition to a cytotoxic agent that is active in the second growth/mitosis phases, $G_{2} / M$, also considers a cytostatic agent that slows down the progression of cells during the synthesis phase $S$. In this case, the optimal administration of the cytotoxic agent is at maximum allowable dose rates (in agreement with the MTD paradigm in medicine) and the blocking agent is used to enhance the action of the killing agent $[24,26,47,48]$.

\subsection{A 3-compartment model with cytotoxic and cytostatic agents}

Mathematically, this situation can be described by a 3-compartment model with the compartments representing the cancer cells in the first growth phase $G_{1}$ (which is lumped with the dormant cells), synthesis $S$, and the second growth phase and mitosis, $G_{2} / M$ [47]. The variables $N_{1}, N_{2}$ and $N_{3}$ denote the average number of cancer cells in the respective compartments, $N=\left(N_{1}, N_{2}, N_{3}\right)^{T}$, and the state space is the first orthant in $\mathbb{R}^{3}$. Transitions of cells through the phases of the cell cycle vary, especially for malignant cancer cells, and follow some empirical distribution. Thus the underlying model is a stochastic process with the individual cells determining the sample paths from cell birth to cell death in cell division. Here, the simplest form, an exponential distribution, is used to model these transitions and this gives a system of linear ordinary differential equations that describe the inflows and outflows between the compartments. Since the outflows of the first and second compartments equal the inflows into the second and third compartment, in the absence of external stimuli the corresponding balance equations simply take the form

$$
\begin{aligned}
& \dot{N}_{2}(t)=-a_{2} N_{2}(t)+a_{1} N_{1}(t), \\
& \dot{N}_{3}(t)=-a_{3} N_{3}(t)+a_{2} N_{2}(t),
\end{aligned}
$$

with $a_{i}$ the inverse mean transit time through the ith compartment. But in the third compartment cell division needs to be taken into account and thus, while the outflow is still given by $a_{3} N_{3}(t)$, the inflow into the first compartment doubles giving

$$
\dot{N}_{1}(t)=-a_{1} N_{1}(t)+2 a_{3} N_{3}(t) .
$$

Following the log-kill hypothesis, under drug actions the number of cells killed or blocked is proportional to both the tumor volume and the drug's concentration. For simplicity, in this model the drug dose rate is identified with the drug's concentration in the plasma. Indeed, the same qualitative results remain valid if, more generally, standard linear pharmacokinetic models are included [26]. The main control is the cytotoxic agent and we denote its dose rate/concentration by $u=u(t)$. The concentration $u(t)$ kills a fraction of the outflow $a_{3} N_{3}(t)$ of cells from the $G_{2} / M$ compartment and thus the number of cells killed is given by $\varphi u(t) a_{3} N_{3}(t)$ with $\varphi$ a constant chemotherapeutic killing parameter. The control set is a compact interval $\left[0, u_{\max }\right]$ with $u_{\max }$ denoting the maximum dose rate/concentration. In the model, the 
control $u$ always appears in conjunction with the constant $\varphi$ and thus, in order to keep the number of free parameters to a minimum, it is combined with the maximum dose rate into one quantity still denoted by $u_{\max }$, but assuming that $u_{\max } \leq 1$. If the concentration is high enough, then indeed $u_{\max }=1$ is realistic: almost all the cancer cells in that compartment can be killed. Cells that are killed in $G_{2} / M$ are counted as outflows from the third compartment, but no longer enter the first compartment. Only the remaining fraction $(1-u) a_{3} N_{3}$ undergoes cell division. Thus, under treatment the first equation changes to

$$
\dot{N}_{1}(t)=-a_{1} N_{1}(t)+2(1-u(t)) a_{3} N_{3}(t) .
$$

The cytostatic blocking agent is applied to slow down the transit times of cancer cells during the synthesis phase $S$ and, as a result, the flow of cancer cells from the second into the third compartment is reduced by a factor of $v(t)$ percent from its original flow of $a_{2} N_{2}(t)$ to $(1-v(t)) a_{2} N_{2}(t), 0 \leq v(t) \leq v_{\max }<1$. This factor $v$ represents a second control in the model with $v(t) \equiv 0$ corresponding to the case when no drug is administered and $v_{\max }$ giving the maximum reductions with full dose. Thus the second and third equation become

$$
\begin{aligned}
& \dot{N}_{2}(t)=-(1-v(t)) a_{2} N_{2}(t)+a_{1} N_{1}(t), \\
& \dot{N}_{3}(t)=-a_{3} N_{3}(t)+(1-v(t)) a_{2} N_{2}(t) .
\end{aligned}
$$

Overall, the dynamics is described by a multi-input bilinear control system of the form

$$
\dot{N}=\left(A+u B_{1}+v B_{2}\right) N
$$

with the matrices given by

$$
A=\left(\begin{array}{ccc}
-a_{1} & 0 & 2 a_{3} \\
a_{1} & -a_{2} & 0 \\
0 & a_{2} & -a_{3}
\end{array}\right), \quad B_{1}=\left(\begin{array}{ccc}
0 & 0 & -2 a_{3} \\
0 & 0 & 0 \\
0 & 0 & 0
\end{array}\right) \quad \text { and } \quad B_{2}=\left(\begin{array}{ccc}
0 & 0 & 0 \\
0 & a_{2} & 0 \\
0 & -a_{2} & 0
\end{array}\right) .
$$

The controls $u$ and $v$ take values in compact intervals $\left[0, u_{\max }\right]$ and $\left[0, v_{\max }\right]$, respectively, with $u_{\max } \leq 1$ and $v_{\max }<1$. It follows that all the matrices $A+u B_{1}+v B_{2}$ have negative diagonal and nonnegative off-diagonal entries, i.e., are so-called $\mathcal{M}$-matrices,

$$
A+u B_{1}+v B_{2}=\left(\begin{array}{ccc}
-a_{1} & 0 & 2(1-u) a_{3} \\
a_{1} & -(1-v) a_{2} & 0 \\
0 & (1-v) a_{2} & -a_{3}
\end{array}\right) \in \mathcal{M} .
$$

From this property, it is immediately seen that the positive octant

$$
\mathbb{P}=\mathbb{R}_{+}^{3}=\left\{N \in \mathbb{R}^{3}: N_{i}>0 \text { for } i=1,2,3\right\}
$$

is positively invariant for the associated control system.

Proposition 2.1. [24] Given arbitrary Lebesgue measurable functions $u:[0, T] \rightarrow\left[0, u_{\max }\right]$ and $v:$ $[0, T] \rightarrow\left[0, v_{\max }\right], u_{\max } \leq 1$ and $v_{\max }<1$, for any positive initial condition $N(0) \in \mathbb{P}$, the solution to the system (2.7) exists over the full interval $[0, T]$ and the values of $N$ remain positive, $N(t) \in \mathbb{P}$ for all $t \in[0, T]$.

\subsection{Steady-state behavior of the proportions in the compartments}

One important and medically realistic consequence of this model is that there exists a well-defined steady state for the proportions of cells in the individual compartments for the uncontrolled model. Let $C(t)=$ $N_{1}(t)+N_{2}(t)+N_{3}(t)$ be the total number of cancer cells and denote the proportions of cancer cells in the three compartments by $x, y$ and $z$, respectively,

$$
x(t)=\frac{N_{1}(t)}{C(t)}, \quad y(t)=\frac{N_{2}(t)}{C(t)} \quad \text { and } \quad z(t)=\frac{N_{3}(t)}{C(t)} .
$$


A direct computation verifies that

$$
\begin{aligned}
& \dot{x}=-a_{1} x+2 a_{3} z-a_{3} x z, \\
& \dot{y}=a_{1} x-a_{2} y-a_{3} y z, \\
& \dot{z}=a_{2} y-a_{3} z-a_{3} z^{2},
\end{aligned}
$$

and, because of the trivial relation $x(t)+y(t)+z(t) \equiv 1$, one of these equations is redundant.

Theorem 2.2. [26] The unit simplex $\Sigma=\{(x, y, z): 0 \leq x, 0 \leq y, 0 \leq z, x+y+z=1\}$ is positively invariant under the dynamical system given by Eqs. (2.9)-(2.11). There exists a unique, asymptotically stable equilibrium point $\left(x_{*}, y_{*}, z_{*}\right)$ that lies in the interior of $\Sigma$ and contains the entire simplex $\Sigma$ in its region of attraction, i.e., given an arbitrary initial condition $\left(x_{0}, y_{0}, z_{0}\right) \in \Sigma$, the solution of Eqs. (2.9)-(2.11) exists for all times $t \geq 0$, lies in $\Sigma$, and converges to $\left(x_{*}, y_{*}, z_{*}\right)$ as $t \rightarrow \infty$.

Consequently, when chemotherapy starts, the system will have specific fractions of cycling cells in the compartments independent of the tumor size. The limiting fractions $\left(x_{*}, y_{*}, z_{*}\right)$ are determined by the coefficients that define the cell cycle kinetics and it is easy to compute these fractions. Conversely, the cell cycle parameters $a_{i}, i=1,2,3$, are functions of these steady-state proportions and the tumor doubling time, i.e, data that can be determined experimentally.

Corollary 2.3. [26] If $T$ denotes the tumor doubling time and $x_{*}, y_{*}$ and $z_{*}$ are the steady-state proportions of cells in the cell cycle compartments $G_{0} / G_{1}, S$ and $G_{2} / M$, respectively, then we have that

$$
a_{3}=\frac{\ln 2}{T} \frac{1}{z_{*}}, \quad a_{2}=\frac{\ln 2}{T} \frac{1+z_{*}}{y_{*}} \quad \text { and } \quad a_{1}=\frac{\ln 2}{T}\left(\frac{2}{x_{*}}-1\right) .
$$

\subsection{Chemotherapy as an optimal control problem}

When chemotherapy is considered as an optimal control problem, an objective function to be minimized is artificially imposed from the outside. Within some degree of approximation, the dynamics represents the underlying biology of the problem. But for the choice of the objective there exist various options with not necessarily a clear "best" choice. In engineering problems, it is quite common to vary the forms of the objective and eventually pick one that induces a satisfactory system response, possibly because it shows good properties (like stability, robustness, etc.) with respect to other design criteria that were not part of the modeling. In other fields, like economics, this may not be appropriate and the objective is clearly specified, for example, if the aim is to minimize the cost of some production process. For problems in the life sciences, the truth lies somewhere in between these two extremes. Clearly, the aim is to cure the patient, but there are many ways to translate this into a mathematical objective.

For chemotherapy, one way or the other, the objective will include a minimization of the overall number of cancer cells. But also the toxicity of the treatment needs to be considered. In principle, this could be done directly by adding as a separate compartment healthy cells (e.g., the bone marrow) and limiting the damage done to this compartment. Alternatively, and this is the approach pursued here, a measure for the side effects of the treatment is included as a penalty term in the objective. Since we are employing the log-kill hypothesis in the evaluation of the damage done to cells, the only consistent structure is to include the total overall doses of the drugs given, $\int_{0}^{T} u(t) d t$ and $\int_{0}^{T} v(t) d t$, with penalties in the objective function. These integrals represent the cumulative toxic side effects of treatment. In the literature also other functional forms of the controls are used, but it becomes questionable how they conform with the underlying biological model. Thus we consider an objective function of the following form

$$
J=r N(T)+\int_{0}^{T}\left(q N(t)+s_{1} u(t)+s_{2} v(t)\right) d t \rightarrow \min
$$

where $r=\left(r_{1}, r_{2}, r_{3}\right)$ and $q=\left(q_{1}, q_{2}, q_{3}\right)$ are row vectors of positive weights. The penalty term

$$
r N(T)=r_{1} N_{1}(T)+r_{2} N_{2}(T)+r_{3} N_{3}(T)
$$


represents a weighted average of the total number of cancer cells at the end of an assumed fixed therapy interval $[0, T]$ and the Lagrangian term

$$
q N(t)=q_{1} N_{1}(t)+q_{2} N_{2}(t)+q_{3} N_{3}(t)
$$

is a running cost that measures the tumor volume during treatment. The positive coefficients $s_{1}$ and $s_{2}$ provide relative weights for the severity of the side effects of the chemotherapeutic agents. In our situation we typically have that $0<s_{2} \ll s_{1}$. Overall, we consider the following optimal control problem:

[C-hom] For a fixed therapy horizon $[0, T]$, minimize the objective (2.12) over all Lebesgue-measurable functions $u:[0, T] \rightarrow\left[0, u_{\max }\right]$ and $v:[0, T] \rightarrow\left[0, v_{\max }\right], u_{\max } \leq 1$ and $v_{\max }<1$, subject to the dynamics (2.4)-(2.6) with initial condition $N(0)=C_{0}\left(x_{*}, y_{*}, z_{*}\right)^{T}$ and $C_{0}$ an estimate for the overall initial tumor size.

\subsection{Structure of optimal controls}

Necessary conditions for optimality are given by the Pontryagin maximum principle [39]. (For some more recent texts on the subject, see $[4,41])$. For this model, since there are no constraints on the terminal state $N(T)$, without loss of generality we define the Hamiltonian function in the form

$$
H=q N+s_{1} u+s_{2} v+\lambda\left(A+u B_{1}+v B_{2}\right) N
$$

with $\lambda$ a multiplier corresponding to the dynamics. If $\left(u_{*}, v_{*}\right)$ are optimal controls, then it follows that there exists an absolutely continuous function $\lambda$ which we write as a row-vector, $\lambda:[0, T] \rightarrow\left(\mathbb{R}^{3}\right)^{*}$, that satisfies the adjoint equation

$$
\dot{\lambda}(t)=-q-\lambda(t)\left(A+u_{*}(t) B_{1}+v_{*}(t) B_{2}\right), \quad \lambda(T)=r,
$$

such that the optimal controls minimize $H$ pointwise over the control set $\left[0, u_{\max }\right] \times\left[0, v_{\max }\right]$ along $\left(\lambda(t), N_{*}(t)\right)$.

Since the dynamics is described by matrices $A+u B_{1}+v B_{2} \in \mathcal{M}$, it also follows that the positive orthant $\mathbb{P}^{*}=\left\{\lambda \in\left(\mathbb{R}^{3}\right)^{*}: \lambda_{i}>0\right.$ for $\left.i=1,2,3\right\}$ in the dual space $\left(\mathbb{R}^{3}\right)^{*}$ is negatively invariant for the adjoint equation [48]. Hence we have the following result:

Proposition 2.4. [24] The solution $\lambda(t)$ to (2.14) exists on all of $[0, T]$ and all components are positive.

Note that the Hamiltonian $H$ is linear in the controls and that the control set is a product of intervals. Hence the minimization of $H$ over the control set reduces to two separate 1-dimensional problems of minimizing a linear function over an interval. If the coefficient multiplying the control is nonzero, the minimum is attained at the boundary points (bang controls), but intermediate values (singular controls) are possible if this function vanishes over some interval. This leads to the following definition of the switching functions for the controls $u$ and $v$,

$$
\Phi_{1}(t)=s_{1}+\lambda(t) B_{1} N_{*}(t) \quad \text { and } \quad \Phi_{2}(t)=s_{2}+\lambda(t) B_{2} N_{*}(t)
$$

and optimal controls satisfy

$$
u_{*}(t)=\left\{\begin{array}{ll}
0 & \text { if } \Phi_{1}(t)>0, \\
u_{\max } & \text { if } \Phi_{1}(t)<0,
\end{array} \quad \text { and } \quad v_{*}(t)= \begin{cases}0 & \text { if } \Phi_{2}(t)>0, \\
v_{\max } & \text { if } \Phi_{2}(t)<0,\end{cases}\right.
$$

with singular controls possible if the corresponding switching function vanishes over an open interval. Explicitly, for this model the switching functions are

$$
\Phi_{1}(t)=s_{1}-2 a_{3} \lambda_{1}(t) N_{3}(t) \quad \text { and } \quad \Phi_{2}(t)=s_{2}+a_{2}\left\{\lambda_{2}(t)-\lambda_{3}(t)\right\} N_{2}(t) .
$$


If $\tau$ is a zero for one of the switching functions $\Phi_{i}$ and we have that $\frac{d \Phi_{i}}{d t}(\tau) \neq 0$, then the switching function $\Phi_{i}$ changes sign at $\tau$ and the control is discontinuous with a switch between the boundary values of the control interval. Controls which only have switchings of this type are called bang-bang. In the other extreme, if an optimal control $u_{*}$ or $v_{*}$ is singular on an open interval $I$, then the corresponding switching function $\Phi_{i}$ and all its derivatives vanish on $I$. Differentiating the switching functions using the dynamics until the controls explicitly appear typically allows to compute the singular controls. However, additional necessary conditions for optimality need to be satisfied that distinguish between locally minimizing and maximizing controls. These are the so-called generalized Legendre-Clebsch conditions for a single control as well as the Goh-condition in case of multiple singular controls (e.g., see [4, 41]). Using these necessary conditions for optimality, for the model [C-hom] singular controls generally can be excluded from optimality. More precisely, we have the following two results:

Theorem 2.5. [24] If $\left(N_{*}, u_{*}, v_{*}\right)$ is an optimal controlled trajectory for problem [C-hom], then there does not exist an interval on which the control $v_{*}$ is singular.

Theorem 2.6. If $\left(N_{*}, u_{*}, v_{*}\right)$ is an optimal controlled trajectory for problem [C-hom], then, for values of the parameters $a_{1}, a_{2}$ and $a_{3}$ in an open and dense set there does not exist an interval on which the control $u_{*}$ is singular. For example, this is satisfied if

$$
\min \left\{a_{1}-2 a_{3}, a_{3}-2 a_{1}\right\}+a_{2}\left(1-v_{\max }\right) \geq 0 .
$$

Proving these results, especially Theorem 2.6, requires some at times elaborate computations since singular controls for the cytotoxic agent are of higher order. At the same time, this fact makes their existence highly unlikely. Condition (2.17) only provides a simple and often easily verified sufficient condition for singular controls not to be optimal, but it is not necessary. Based on these theoretical results, generically optimal controls are bang-bang.

\subsection{Numerical examples}

We give a couple of numerical solutions for the parameter values $a_{1}=0.197, a_{2}=0.395$ and $a_{3}=0.107$ taken from [47] and the control limits $u_{\max }=0.95$ and $v_{\max }=0.30$. For these parameter values, condition (2.17) is satisfied and singular controls are not optimal. Figure 1 shows two qualitatively different scenarios of locally optimal controls $u$ and $v$ and their corresponding trajectories. Second order optimality conditions for flows of bang-bang extremals can be verified along the lines outlined in $[41,42]$ and both of these numerically computed computed controlled trajectories are strong local minima.

In the graphs on the left, the weights at the cancer cells are all taken to be equal, $r=(1,1,1)$ and $q=(1,1,1)$, and in this case the optimal control $u$ starts with a segment along which we have $u \equiv u_{\max }$. It would make no sense to block the flow of cells while a cytotoxic agent is active and accordingly the cytostatic agent is inactive at the beginning. It only becomes activated between the switching times $t_{1}=10.115$ and $t_{3}=19.835$; the cytotoxic agent is stopped at time $t_{2}=11.054$. It is thus only turned on shortly before the killing agent is withdrawn which corresponds to the timing effects in the cell cycle. After the cytotoxic agent is withdrawn, then the cytostatic agent is active for most of the time. This simply is an alternative mechanism to slow down the growth of the tumor. If one views the whole therapy interval as one coherent unit and only looks at the total number of cancer cells, $C(t)=N_{1}(t)+N_{2}(t)+N_{3}(t)$, then $C$ decreases from $C(0)=1$ to $C(21)=0.868$.

In the second example, the weights are skewed to make it more important to minimize the value of the cancer cells at the final time, $r=(8.25,8.25,8.25)$, and we relaxed the weight on the intermediate size of the tumor, $q=(0.1,0.1,0.1)$. This leads to a shift of the interval where the cytotoxic agent is active towards the end of therapy and now the cytostatic agent is active at the beginning. In fact, and according with the dynamics of the cell cycle, it is withdrawn shortly before the cytotoxic agent becomes active. For these weights, we only see a reduction of the total tumor numbers from $C(0)=1$ to $C(21)=0.978$, basically just a maintenance of the current tumor volume. However, the total amount of cytotoxic agents 

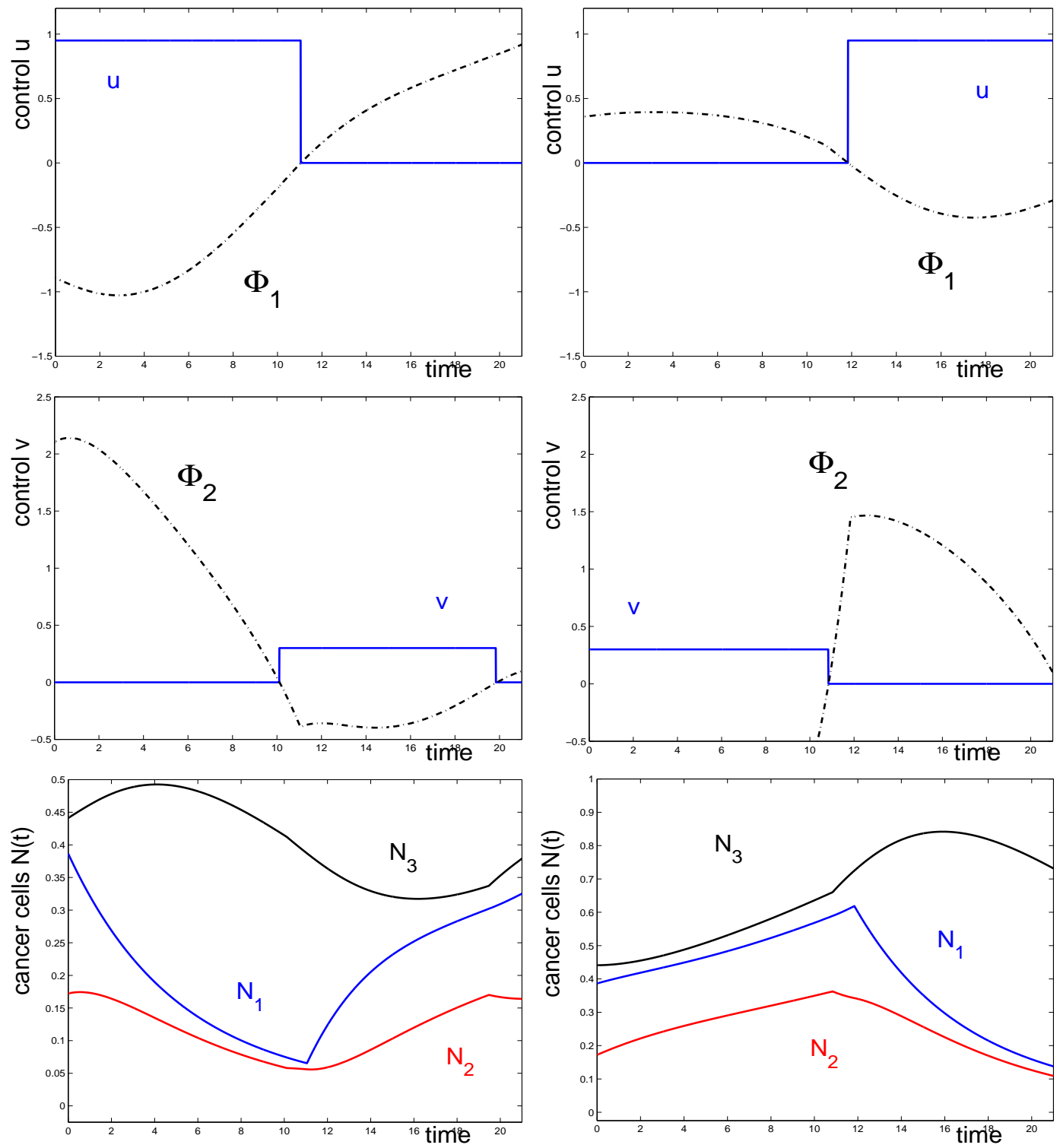

FiguRE 1. Examples of locally optimal controls $u$ (cytotoxic agent, top), $v$ (cytostatic agent, middle) and their corresponding trajectories (bottom) for different weights $r=$ $(1,1,1)$ and $q=(1,1,1)$ on the left and $r=(8.25,8.25,8.25)$ and $q=(0.1,0.1,0.1)$ on the right from the steady-state solution.

used is lower under this scenario, 9.165 versus 11.054 in the first case. Clearly, these tumor kill numbers can be increased by allowing for higher side effects, i.e., by using a lower value for $s_{1}$. The numbers used here only serve to illustrate the general mechanisms. But overall the upfront dosing scheme for the cytotoxic agent leads to better tumor reductions and thus this objective functional would probably be preferred in practice. 


\section{Heterogeneous Tumor Populations: Lower Dose Rate Protocols}

If the tumor is a heterogeneous agglomeration of subpopulations of various sensitivities, optimal administration of cytotoxic agents no longer needs to be bang-bang. It seems rather obvious that, as resistance builds up, full dose therapies may do more harm to the healthy cells than have beneficial effects and an analysis of various mathematical models for developing resistance under chemotherapy confirm this fact (e.g., $[25,50])$. More interestingly, and less obvious a priori, it also holds that when the tumor simply consists of subpopulations of different chemotherapeutic sensitivities, then lower dose rate administrations that vary the concentrations of the agents (according to singular dose rates) become viable candidates for optimality (with implied local optimality properties) from the beginning.

\subsection{A mathematical model with three subpopulations}

In a recent paper, Green, Lovi, Gottesman and Levy [11] formulate a mathematical model for multi-drug resistance in cancer which, starting with a continuum of drug resistance levels described by a compact interval, shows that, as a response to cell density and mutations over time, out of this continuum a finite number of specific traits (resistance levels) emerge to become dominant while other resistance levels diminish. In view of this result, it is thus of interest to analyze models that have specific levels of chemotherapeutic sensitivities of the tumor populations. Here we review some mathematical results obtained in [27] about such a model for a single chemotherapeutic agent when we distinguish three such levels calling the subpopulations "sensitive", $S$, "partially sensitive", $P$, and "resistant", $R$. But the terminology is only meant to indicate that these populations have different sensitivities towards a chemotherapeutic agent with $S$ the highest and $R$ the lowest. As more levels of sensitivity are taken into account in the model, lower time-varying dose rates, as they are given by singular controls, become a treatment option immediately while this is only the case once a significant residuum of resistant cells has been created in simpler 2-compartment models [25].

The underlying mathematical model is that of an ergodic Markov chain analogous to the one formulated by Hahnfeldt, Folkman and Hlatky in [14], but for three subpopulations. We assume that these subpopulations grow at separate growth rates $\alpha_{1}, \alpha_{2}$ and $\alpha_{3}$, but do not make assumptions on the order of the growth rates. Transitions between all compartments are allowed and assumed. We denote the transition rates from the sensitive to the partially sensitive and resistant compartments by $\sigma_{P}$ and $\sigma_{R}$, respectively, and use analogous notations for the other transition rates. Thus, for example $\rho_{P}$ denotes the transition rates from resistant to partially sensitive cells. These rates are assumed to be constant and positive. This generates an ergodic structure in which all compartments are repeatedly visited by cells. As above, cell kill by a chemotherapeutic agent is expressed by the standard linear log-kill hypothesis. Denoting the concentration of the drug in the bloodstream by $u$, the rate of cells eliminated is given by $\varphi_{i} u, i=1,2,3$, with the coefficients $\varphi_{1}, \varphi_{2}$ and $\varphi_{3}$ representing the effectiveness of the drug on the sensitive, partially sensitive and resistant subpopulations, respectively. Thus $\varphi_{1}>\varphi_{2}>\varphi_{3} \geq 0$. The case $\varphi_{3}=0$ corresponds to the situation of a fully resistant subpopulation $R$. The controlled dynamics is determined by the inflows and outflows from the various compartments and is given by the following 3-dimensional linear system of equations:

$$
\begin{aligned}
& \dot{S}=\left(\alpha_{1}-\sigma_{P}-\sigma_{R}-\varphi_{1} u\right) S+\pi_{S} P+\rho_{S} R, \\
& \dot{P}=\sigma_{P} S+\left(\alpha_{2}-\pi_{S}-\pi_{R}-\varphi_{2} u\right) P+\rho_{P} R, \\
& \dot{R}=\sigma_{R} S+\pi_{R} P+\left(\alpha_{3}-\rho_{S}-\rho_{P}-\varphi_{3} u\right) R .
\end{aligned}
$$

Without loss of generality, we assume that all initial conditions $S_{0}, P_{0}$ and $R_{0}$ are positive. Admissible controls are Lebesgue measurable functions with values in a compact interval $\left[0, u_{\max }\right], u:[0, T] \rightarrow$ $\left[0, u_{\max }\right], t \mapsto u(t)$, and once more it is easy to see that for any admissible control $u$, the solution to equations (3.1)-(3.3) exists on the full interval $[0, T]$ and all components are positive. 


\subsection{Steady-state behavior of the relative proportions}

As in the cell-cycle specific model for a homogeneous population, the balance equations are linear and thus the proportions of the respective populations, i.e.,

$$
x=\frac{S}{S+P+R}, \quad y=\frac{P}{S+P+R}, \quad \text { and } \quad z=\frac{R}{S+P+R},
$$

obey Riccati differential equations. If we denote the relative growth rates of the subpopulations by

$$
\nu_{s}=\alpha_{1}-\sigma_{P}-\sigma_{R} \quad \text { and } \quad \hat{\nu}_{s}=\alpha_{1}-\sigma_{P}-\sigma_{R}-\varphi_{1} u
$$

and analogously for $P$ and $R$, then a direct computation verifies that

$$
\begin{aligned}
& \dot{x}=\nu_{S} x+\pi_{S} y+\rho_{S} z-x\left(\alpha_{1} x+\alpha_{2} y+\alpha_{3} z\right), \\
& \dot{y}=\sigma_{P} x+\nu_{P} y+\rho_{P} z-y\left(\alpha_{1} x+\alpha_{2} y+\alpha_{3} z\right), \\
& \dot{z}=\sigma_{R} x+\pi_{R} y+\nu_{R} z-z\left(\alpha_{1} x+\alpha_{2} y+\alpha_{3} z\right) .
\end{aligned}
$$

As before, let $\Sigma$ denote the unit simplex in $\mathbb{R}^{3}$, i.e., $\Sigma=\{(x, y, z): x \geq 0, y \geq 0, z \geq 0, x+y+z=1\}$. Because of the multitude of free parameters in the model, the existence of a unique equilibrium point inside $\Sigma$ now becomes more difficult to establish, but still holds. The result below then is a direct application of Poincaré-Bendixson theory.

Proposition 3.1. [27] The unit simplex $\Sigma$ is positively invariant under the dynamics (3.4)-(3.6) and contains exactly one equilibrium point $\left(x_{*}, y_{*}, z_{*}\right)$. If there exists a trajectory starting in the boundary of $\Sigma$ that converges to $\left(x_{*}, y_{*}, z_{*}\right)$, then the equilibrium point is asymptotically stable and contains the entire simplex $\Sigma$ in its region of attraction.

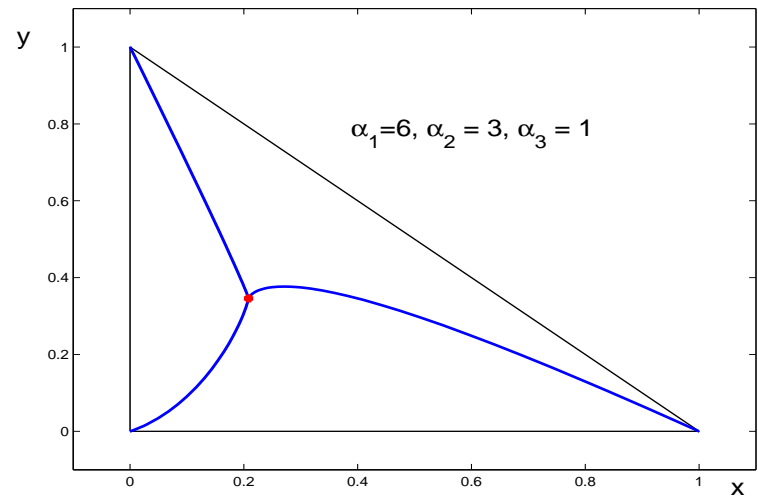

FiguRE 2. Equilibrium point and trajectories from the vertices of the unit simplex $\Sigma$.

We illustrate the typical geometric structure of the phase portrait in Fig. 2. We have chosen the transit rates as $\sigma_{P}=4, \sigma_{R}=2, \pi_{S}=1, \pi_{R}=2, \rho_{S}=0.5$ and $\rho_{P}=0.25$ and the growth rates for the respective compartments are $\alpha_{1}=6, \alpha_{2}=3$ and $\alpha_{3}=1$. The numbers are just for illustration, but in this scenario the sensitive cells are the most strongly proliferating ones and the resistant population is the slowest growing subpopulation which would be consistent with the Norton-Simon hypothesis. 


\subsection{Singular controls}

As above, we consider the optimal control problem to minimize the tumor burden over a fixed therapy interval $[0, T]$ through administration of chemotherapy with the toxicity of treatment added as a penalty term to the objective.

[C-het] For a fixed therapy horizon $[0, T]$, minimize the objective

$$
J(u)=r N(T)+\int_{0}^{T}(q N(t)+u(t)) d t \rightarrow \min
$$

over all Lebesgue-measurable functions $u:[0, T] \rightarrow\left[0, u_{\max }\right]$ subject to the dynamics (3.1)-(3.3).

In the objective, we again denote the state of the system by $N, N=(S, P, R)^{T}$, written as a column vector, and the coefficients $r=\left(r_{1}, r_{2}, r_{3}\right)$ and $q=\left(q_{1}, q_{2} . q_{3}\right)$ are positive weights which we write as row vectors. Without loss of generality, we normalized the weight at the control to be 1 .

Necessary conditions for optimality again are given by the Pontryagin maximum principle. Here the dynamics is a single-input bilinear control system of the form

$$
\dot{N}=(A+u B) N
$$

with the matrices $A$ and $B$ given by

$$
A=\left(\begin{array}{ccc}
\alpha_{1}-\sigma_{P}-\sigma_{R} & \pi_{S} & \rho_{S} \\
\sigma_{P} & \alpha_{2}-\pi_{S}-\pi_{R} & \rho_{P} \\
\sigma_{R} & \pi_{R} & \alpha_{3}-\rho_{S}-\rho_{P}
\end{array}\right) \quad \text { and } \quad B=\left(\begin{array}{ccc}
-\varphi_{1} & 0 & 0 \\
0 & -\varphi_{2} & 0 \\
0 & 0 & -\varphi_{3}
\end{array}\right)
$$

and the adjoint equation takes the form

$$
\dot{\lambda}(t)=-q-\lambda(t)\left(A+u_{*}(t) B\right), \quad \lambda(T)=r .
$$

Proposition 3.2. [27] All multipliers $\lambda_{i}, i=1,2,3$, are positive over the interval $[0, T]$.

If $u_{*}:[0, T] \rightarrow\left[0, u_{\max }\right]$ is an optimal control with corresponding trajectory $N_{*}$, then there exists a solution $\lambda=\left(\lambda_{1}, \lambda_{2}, \lambda_{3}\right):[0, T] \rightarrow\left(\mathbb{R}^{3}\right)^{*}$ of the terminal value problem (3.9) such that, with the Hamiltonian function $H$ given by

$$
H=q N+u+\lambda(A+u B) N,
$$

the optimal control at time $t, u_{*}(t)$, minimizes the Hamiltonian $H$ pointwise over the control set $\left[0, u_{\max }\right]$ along $\left(\lambda(t), N_{*}(t), u_{*}(t)\right)$. As before, this leads to the definition of the switching function as

$$
\Phi(t)=1+\lambda(t) B N_{*}(t),
$$

and optimal controls satisfy

$$
u_{*}(t)= \begin{cases}0 & \text { if } \Phi(t)>0 \\ u_{\max } & \text { if } \Phi(t)<0\end{cases}
$$

Contrary to the models for homogeneous populations, here singular controls are viable and optimal controls will be concatenations of bang and singular pieces that need to determined through an analysis of the properties of the switching functions. If the control is singular over an open interval $I$, then (modulo some degenerate, nongeneric situations) the control $u$ explicitly occurs for the first time only in an even numbered derivative of the switching function. A singular control is said to be of intrinsic order $k$ if this is the $2 k$-th derivative. It then is a necessary condition for optimality, the so-called generalized Legendre-Clebsch condition $[4,41]$, that

$$
(-1)^{k} \frac{\partial}{\partial u} \frac{d^{2 k}}{d t^{2 k}} \frac{\partial H}{\partial u}\left(\lambda(t), N_{*}(t), u_{*}(t)\right)=(-1)^{k} \frac{\partial}{\partial u} \Phi^{(2 k)}(t) \geq 0 .
$$


If strict inequality holds in this equation, we say that the strengthened Legendre-Clebsch condition is satisfied. This often is an indication of local optimality properties of the singular control.

If an optimal control is singular over an open interval $I$, then $\lambda(t) B N_{*}(t) \equiv-1$ on this interval. The following simple formula, which follows from a direct calculation, allows us to organize the derivatives of the switching function in a structured manner.

Proposition 3.3. Suppose $M$ is a constant matrix and let $\Psi(t)=\lambda(t) M N(t)$ where $N$ is a solution to the dynamics (3.8) for the control $u$ and $\lambda$ is a solution of the corresponding adjoint equation (3.9). Then

$$
\dot{\Psi}(t)=\lambda(t)[A+u B, M] N(t)-q M N(t),
$$

with $[X, Y]=Y X-X Y$ denoting the commutator of the matrices $X$ and $Y$.

We have chosen the sign of the commutators of the matrices to be consistent with the definition of the Lie bracket $[X, Y]$ of the linear vector fields $X(N)=X N$ and $Y(N)=Y N$.

If the switching function $\Phi$ vanishes identically over an interval $I$, we thus have that

$$
\begin{aligned}
\dot{\Phi}(t) & =\lambda(t)\left[A+u_{*}(t) B, B\right] N_{*}(t)-q B N_{*}(t) \\
& =\{\lambda(t)[A, B]-q B\} N_{*}(t)=0, \\
\ddot{\Phi}(t) & =\lambda(t)\left[A+u_{*}(t) B,[A, B]\right] N_{*}(t)-q[A, B] N_{*}(t)-q B\left(A+u_{*}(t) B\right) N_{*}(t) \\
& =\left\{(\lambda(t)[A,[A, B]]-q[A, B]-q B A)+u_{*}(t)\left(\lambda(t)[B,[A, B]]-q B^{2}\right)\right\} N_{*}(t)=0,
\end{aligned}
$$

with the iterated brackets denoting successive Lie brackets (or commutators) of the matrices $A$ and $B$. The coefficient multiplying the control $u$ in the second derivative of the switching function is given by

$$
\frac{\partial}{\partial u} \frac{d^{2}}{d t^{2}} \frac{\partial H}{\partial u}\left(\lambda(t), N_{*}(t), u_{*}(t)\right)=\left\{\lambda(t)[B,[A, B]]-q B^{2}\right\} N_{*}(t) .
$$

Direct, but somewhat lengthy calculations verify that all entries in the matrix $[B,[A, B]]$ are nonpositive and it follows from the dynamics that all components of the state $N_{*}$ and the multiplier $\lambda$ are positive. Hence $\lambda(t)[B,[A, B]] N_{*}(t) \leq 0$. Furthermore,

$$
q B^{2} N_{*}(t)=q_{1} \varphi_{1}^{2} S_{*}(t)+q_{2} \varphi_{2}^{2} P_{*}(t)+q_{3} \varphi_{3}^{2} R_{*}(t)>0
$$

so that

$$
-\frac{\partial}{\partial u} \frac{d^{2}}{d t^{2}} \frac{\partial H}{\partial u}\left(\lambda(t), N_{*}(t), u_{*}(t)\right)>0 .
$$

Hence we have the following result:

Proposition 3.4. [27] Singular controls are of order 1 and the strengthened Legendre-Clebsch condition for minimality is satisfied.

Solving equation (3.16) for the control gives the following formula for the singular control

$$
u_{\text {sing }}(t)=\frac{\{\lambda(t)[A,[A, B]]-q[A, B]-q B A\} N_{*}(t)}{\left\{-\lambda(t)[B,[A, B]]+q B^{2}\right\} N_{*}(t)} .
$$

While this formula is expressed using the state $N_{*}(t)$, note that it is unchanged if we divide both numerator and denominator by the total number of cancer cells and thus could also be written in terms of the proportions $x, y$ and $z$. However, the multiplier $\lambda$ implicitly depends on the actual states. In order to be admissible, the values need to lie in the control set $\left[0, u_{\max }\right]$. It follows from the strengthened Legendre-Clebsch condition that the denominator is positive. In the numerator, computations of the relevant matrices verify that all component of the vector $-q B A$ are positive. There exist coefficients in the matrices $[A,[A, B]]$ and the vector $-q[A, B]$ that are negative, but they are far outweighed by 
positive entries. Thus generally, and this is what we have consistently seen in numerical computations, the values of the expression (3.17) are positive and thus are admissible for suitable upper bounds $u_{\max }$.

Along a singular arc, the multiplier $\lambda$ is determined up to one degree of freedom by the conditions $\Phi(t)=1+\lambda(t) B N_{*}(t) \equiv 0$ and $\dot{\Phi}(t)=\{\lambda(t)[A, B]-q B\} N_{*}(t) \equiv 0$. But only solutions with positive entries give rise to singular controls. Yet, in principle, for most states there exists a 1-parameter family of singular arcs through this point. This makes analyzing optimal concatenations between bang and singular controls difficult and this analysis still needs to be completed. Below we include one example of a controlled trajectory that includes a singular arc and satisfies the necessary conditions for optimality of the maximum principle. As it can be seen, for these numerical values the dose rates are reduced to about $60 \%$ along the singular segment.
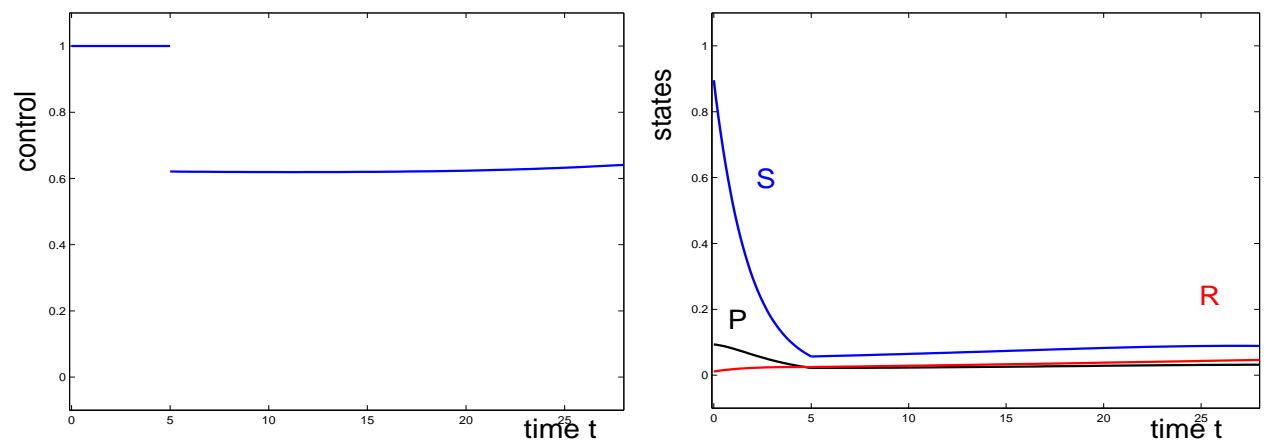

FIGURE 3. Control and states for a bang-singular controlled trajectory.

\section{Cancer Chemotherapy under Immune System Interactions: Chemo-Switch Protocols}

In the previous sections, the administration of chemotherapy was considered for mathematical models that confine themselves to tumor growth. A natural and important medical question is to what extent results about the optimal administration schedules change as various aspects of the tumor microenvironment are incorporated into the modeling. One major component here is the immune system with both beneficial and detrimental effects. The purpose of the immune system is to protect the organism from disease. In order to fulfill this function, it needs to be able to detect a wide variety of agents, from viruses to bacteria to parasites, but also must be able to distinguish these from the organism's own healthy tissue in order not to harm the organism. Since the immune system's first response to its environment is on the basis of a discrimination between "own" and "foreign" objects, tumor cells will be tolerated by the patient's immune system if, essentially, they are classified as "own" cells [33]. However, tumor cells generally exhibit a large number of abnormalities (such as mutated proteins, under- or over-expressed normal proteins and many more) that lead to the appearance of specific antigens that will be classified as "foreign" and thus do trigger reactions by both the innate and adaptive immune system that are able to control small tumors, so-called immunesurveillance $[17,45]$. The competitive interaction between tumor cells and the immune system is complex and involves a large number of events with the kinetics of the interplay strongly nonlinear and the possible outcome of this interplay is not only constituted by either tumor suppression or tumor outbreak. For example, it has been hypothesized that in case of a fully developed and metastatic tumor, upregulation of the immune system caused by the tumor may be responsible for controlling small metastases. Also, there exist theoretical immuno-oncologic studies that were largely inferred from clinical data and come to the conclusion that in some cases the immune system may be able to keep the tumor in a dynamic equilibrium that corresponds to a microscopic 
(undetectable) dormant state [29-31], so-called tumor dormancy. Thus, there exist beneficial effects that may be exploited in cancer treatment.

\subsection{Mathematical modeling of tumor-immune system interactions}

With the increased understanding of the mechanisms of the immune system in connection with AIDS (acquired immune deficiency syndrome) research, in recent years the literature on the mathematical description of tumor immune system interactions has seen a strong resurgence, e.g., see $[18,32,37,38]$. In particular, in this context chemotherapy has also been analyzed as optimal control problem by de Pillis and Radunskaya [37]. But these more recent models are quite complex in their detail which makes it difficult to apply the techniques of optimal control and pursue a full analysis of the solutions. Historically, one of the first publications on this topic is the 1980 paper by Stepanova [43] where a mathematical model of two ordinary differential equations has been proposed that aggregates the interactions between cancer cell growth and the activities of the immune system during the development of cancer. Precisely because of its simplicity - a few parameters incorporate many medically important features - the underlying equations have been widely accepted as a basic model. In that model, the main features of tumor immune system interactions are aggregated into just two variables, the tumor volume, $x$, and the immunocompetent cell densities, $y$, a non-dimensional, order of magnitude quantity related to various types of immune cells ( $T$-cells) activated during the immune reaction. Stepanova's model takes the following form:

$$
\begin{aligned}
& \dot{x}=\mu_{C} x F(x)-\gamma x y, \\
& \dot{y}=\mu_{I}(1-\beta x) x y-\delta y+\alpha,
\end{aligned}
$$

with all Greek letters denoting constant coefficients. Equation (4.2) summarizes the main features of the immune system's reaction to cancer: $\alpha$ models a constant rate of influx of $T$-cells generated through the primary organs and $\delta$ is simply the rate of natural death of the $T$-cells. The first term in this equation models the proliferation of lymphocytes. For small tumors it is stimulated by the anti-tumor antigen which is assumed to be proportional to the tumor volume $x$. But large tumors suppress the activity of the immune system and this is expressed through the inclusion of the term $-\beta x^{2}$. Thus $1 / \beta$ corresponds to a threshold beyond which the immunological system becomes depressed by the growing tumor. The coefficients $\mu_{I}$ and $\beta$ are used to calibrate these interactions and in the product with $y$ collectively describe a state-dependent influence of the cancer cells on the stimulation of the immune system. The first equation, (4.1), models tumor growth. The coefficient $\gamma$ denotes the rate at which cancer cells are eliminated through the activity of $T$-cells and the term $\gamma x y$ thus models the beneficial effect of the immune reaction on the cancer volume. Lastly, $\mu_{C}$ is a tumor growth coefficient and $F$ is a functional parameter that allows to specify various growth models for the cancer cells. In Stepanova's original formulation exponential growth of the cancer cells was considered, i.e., $F(x)=F_{E}(x) \equiv 1$, but in several extensions both logistic and Gompertzian models have been used as well [20,51]. Especially, in the paper [20] by Kuznetsov, Makalkin, Taylor and Perelson the model is biologically validated based on in vivo data of B-lymphoma $B C L_{1}$ in the spleen of mice.

Figure 4 shows three phase portraits of the system (4.1)-(4.2) for (a) a classical logistic model, $F_{L}(x) \equiv$ $1-\frac{x}{x_{\infty}}$, (b) a generalized logistic model with $\nu=2, F_{G L}(x)=1-\left(\frac{x}{x_{\infty}}\right)^{2}$, and (c) an exponential growth function, $F_{E}(x) \equiv 1$. The parameter values that were used to generate these figures are summarized in Table 1 and were taken from the paper by Kuznetsov et al. [20]. These values are non-dimensional on an order of magnitude scale with the tumor volume $x$ expressed in terms of multiples of $10^{6}$ cells and $y$ a dimensionless quantity that describes the immuno-competent cell density as an order of magnitude relative to base value 1 . The time scale is taken relative to the tumor cell cycle in mice and is in terms of 0.11 days [20].

For the specified parameter values, the dynamics is multi-stable and the system has both locally asymptotically stable microscopic and macroscopic equilibrium points as well as an unstable saddle point. The tumor volumes are small and the immuno-competent cell densities are upregulated (above 1) for the 

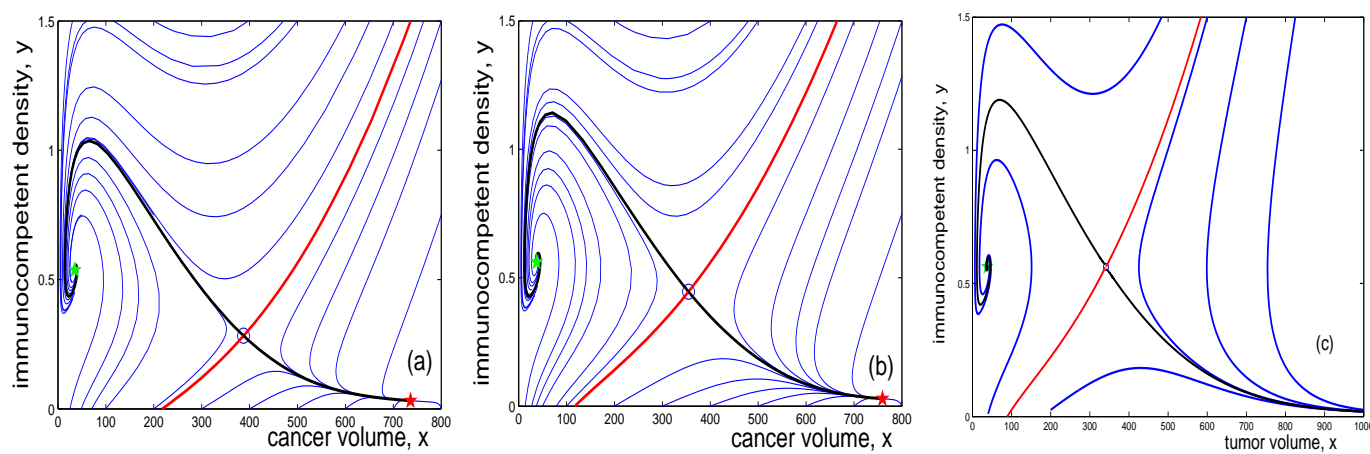

Figure 4. Phase portraits for the system (4.1)-(4.2) with (a) logistic, (b) generalized logistic and (c) exponential growth functions. The parameter values are given in Table 1.

\begin{tabular}{|c|l|c|c|}
\hline $\begin{array}{c}\text { variable } \\
\text { parameters }\end{array}$ & interpretation & numerical value & Reference \\
\hline$x$ & tumor volume & & \\
$y$ & immuno-competent cell density & 0.1181 & {$[20]$} \\
$\alpha$ & rate of influx of T-cells & 0.00264 & {$[20]$} \\
$\beta$ & inverse threshold for tumor suppression & 1 & {$[20]$} \\
$\gamma$ & interaction rate & 0.37451 & {$[20]$} \\
$\delta$ & death rate & 0.5599 & \\
$\mu_{C}$ & tumor growth parameter & 0.00484 & \\
$\mu_{I}$ & tumor stimulated proliferation rate & 780 & \\
$x_{\infty}$ & fixed carrying capacity & & \\
\hline
\end{tabular}

TABLE 1. Variables and parameters used for the phase portraits shown in Fig. 4.

microscopic equilibrium point. This corresponds to a situation when the immune system is able to control the tumor. We denote the corresponding equilibrium point by $\left(x_{b}, y_{b}\right)$ and call it benign. The macroscopic equilibrium point is characterized by more than tenfold higher tumor volumes and basically totally depressed immuno-competent cell densities. In these solutions, the tumor has totally suppressed the immune system and almost reached its carrying capacity. We denote the corresponding equilibrium point by $\left(x_{m}, y_{m}\right)$ and call it malignant. Both equilibria are locally asymptotically stable and the regions of attraction are open and connected sets that we call the benign and malignant regions, respectively. Note that these regions are separated by the stable manifold of the saddle (shown as a red curve in each diagram) which forms the common boundary of these regions. This is a general property of so-called Morse-Smale systems [12].

We can see from the phase portraits that the malignant region is larger for fast growing tumors corresponding to the generalized logistic and exponential models. In fact, for a generalized logistic growth model of the form $F(x)=1-\left(\frac{x}{x_{\infty}}\right)^{\nu}$ with $\nu>0$ and for the parameter values from Table 1, for small enough $\nu$ there only exists one globally asymptotically stable equilibrium which corresponds to a microscopic and thus benign equilibrium point. In this sense, for very slowly growing tumors, in this model the immune system is able to control the disease. However, as $\nu$ increases, an unstable saddle and a stable macroscopic (malignant) equilibrium are born in a saddle-node bifurcation and the benign region decreases at the expense of the malignant regions as the parameter $\nu$ increases. The malignant region increases in size with increasing tumor growth rate converging to the region of attraction for the 
model with exponential growth in the limit $\nu \rightarrow \infty$ reflecting the fact that the immune system becomes increasingly overwhelmed by a fast growing tumor [22].

Overall, tumor-immune system interactions thus exhibit a multitude of dynamic properties that include multi-stability, i.e., persistence of both benign and malignant scenarios. From a practical point of view, the question of curing cancer can thus be related to the mathematical problem of how to move an initial condition that lies in the malignant region into the benign region. This requires therapy and can naturally be formulated and analyzed as an optimal control problem.

\subsection{Chemotherapy protocols for strongly targeted drugs as an optimal control problem}

We only consider chemotherapy with a strongly targeted chemotherapeutic drug, i.e., we assume that the side effects on the immune system are minor and neglect them in the modeling. For sake of specificity, we also choose a Gompertzian growth rate, $F(x)=-\log \left(\frac{x}{x_{\infty}}\right)$. Thus, under the usual log-kill hypothesis, the following dynamics results under chemotherapy:

$$
\begin{array}{ll}
\dot{x}=-\mu_{C} x \log \left(\frac{x}{x_{\infty}}\right)-\gamma x y-\kappa x u, & x(0)=x_{0}, \\
\dot{y}=\mu_{I}\left(x-\beta x^{2}\right) y-\delta y+\alpha, & y(0)=y_{0} .
\end{array}
$$

Writing the state of the system as $z=(x, y)^{T}$, we can express the dynamics in the vector field form

$$
\dot{z}=f(z)+u g(z)
$$

with drift and control vector fields given by

$$
f(z)=\left(\begin{array}{c}
-\mu_{C} x \log \left(\frac{x}{x_{\infty}}\right)-\gamma x y \\
\mu_{I}\left(x-\beta x^{2}\right) y-\delta y+\alpha
\end{array}\right) \quad \text { and } \quad g(z)=\left(\begin{array}{c}
-\kappa x \\
0
\end{array}\right) .
$$

The state space for the system (4.1)-(4.2) is the set $\mathbb{M}=\left\{(x, y): 0<x<x_{\infty}, 0<y\right\}$ and we assume that initial conditions lie in $\mathbb{M}$.

Proposition 4.1. The region $\mathbb{M}$ is positively invariant for the control system, i.e., given an arbitrary admissible control $u:[0, T] \rightarrow[0,1]$ defined over an interval $[0, T], T \leq \infty$, the solution to the dynamics (4.3) and (4.4) exists on $[0, T]$ and the corresponding trajectory lies in $\mathbb{M}$.

The practical aim of therapy is to move an initial state $\left(x_{0}, y_{0}\right)$ of the system that lies in the malignant region of the uncontrolled system into the region of attraction of the stable, benign equilibrium point while keeping side effects tolerable. Here we consider the following optimal control problem:

[C-TI $]$ for a free terminal time $T$, minimize the objective

$$
J=A x(T)-B y(T)+C \int_{0}^{T} u(t) d t+D T,
$$

over all Lebesgue measurable functions $u:[0, T] \rightarrow[0,1]$ subject to the dynamics (4.3) and (4.4),

$$
\begin{array}{ll}
\dot{x}=-\mu_{C} x \log \left(\frac{x}{x_{\infty}}\right)-\gamma x y-\kappa x u, & x(0)=x_{0}, \\
\dot{y}=\mu_{I}\left(x-\beta x^{2}\right) y-\delta y+\alpha & y(0)=y_{0} .
\end{array}
$$


The objective function consists of three separate components: (i) the penalty term $A x(T)-B y(T)$ at the final time is designed to induce the state of the system to move from the malignant into the benign region, (ii) the term $\int_{0}^{T} u(t) d t$ measures the amounts of drugs given and (iii) the penalty term $D T$ on the final time makes the mathematical problem well-posed. All coefficients are positive. We emphasize that the coefficients in the objective (4.6) are variables of choice and typically will be calibrated to tailor the response of the system. The choice of the weights aims at striking a balance between the benefit at the terminal time $T, A x(T)-B y(T)$, and the overall side effects measured by the total amount of drugs given, while it guarantees the existence of an optimal solution by also penalizing the free terminal time $T$. Overall, the coefficients $A, B, C$ and $D$ are variables of choice that can be fine tuned to calibrate the system's optimal response. We discuss the rationale behind each term.

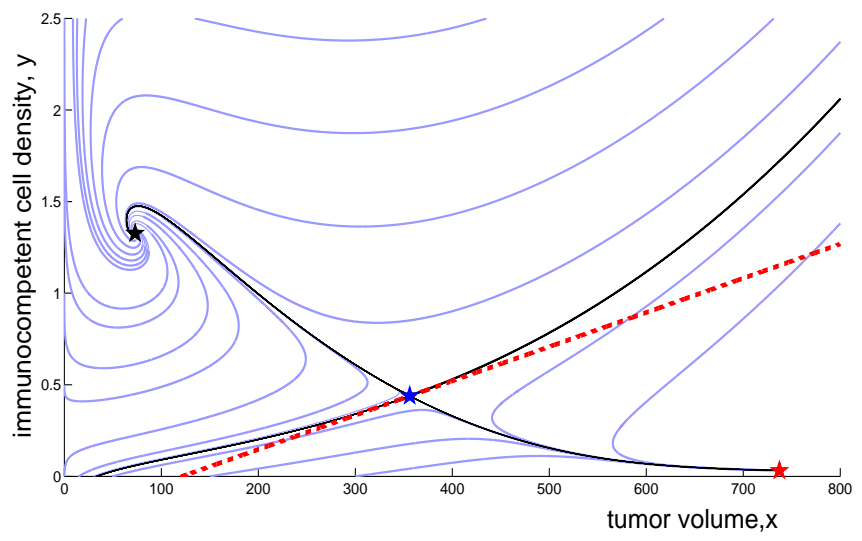

Figure 5. Phase portraits for the system (4.1)-(4.2) with a Gompertzian growth function and parameter values given in Table 1.

(i) The main feature here is to formulate the objective (4.6) in such a way that minimization induces a transfer of the system from the malignant into the benign region of the state space. Figure 5 shows the phase portrait for a Gompertzian growth model and, as there exist small tumor volumes that still lie in the malignant region if the immune system is depressed, a mere minimization of the tumor volume alone may no longer be adequate. (The stable and unstable manifolds of the saddle are shown as the heavy solid curves in Fig. 5). Rather, the geometric shape of the separatrix matters. Ideally, if a functional description of this manifold could be given, one would minimize or maximize the level sets of this function to achieve a transfer into the benign region. But these are generally highly transcendental equations that cannot be solved explicitly. On the other hand, local approximations for the separatrix at the saddle point are easily obtained since the stable eigenspace at the saddle, shown as a dashed red line in Fig. 5, is the tangent space to the separatrix. The normal vector to this tangent line can serve as a reasonable direction in which we want the system to move. Another natural option might be to take the direction of the unstable eigenvector at the saddle since this is the path which uncontrolled trajectories in the benign region will closely follow. Here we pursue the first approach. Let $\mathfrak{v}=(B, A)^{T}$ denote a stable eigenvector $\mathfrak{v}$ of the saddle point $\left(x_{s}, y_{s}\right)$ oriented so that both $A$ and $B$ are positive numbers. It follows from the geometry that $A$ and $B$ will have the same sign and including in the objective a term of the form $A x(T)-B y(T)$ evaluated at the final time $T$ gives the correct direction to minimize in the objective. The level sets of this quantity are lines parallel to the tangent space of the stable manifold of the saddle, and minimizing this quantity thus creates an incentive for the system to move into the benign region.

(ii) As for the models considered earlier, we do not include a separate compartment of healthy cells that would describe the side effects of treatment. These are only indirectly measured through the total 
amounts of drugs given. Therefore, in the objective function to be minimized we once more include the term $\int_{0}^{T} u(t) d t$ as soft constraint.

(iii) The last term in the objective function, which can be written either under the integral or as a separate penalty term $D T$, is included to give a mathematically well-posed problem formulation. The reason is that the existence of the asymptotically stable, benign equilibrium point generates controlled trajectories that improve the value $A x(T)-B y(T)$ of the objective along the trivial control $u=0$. If no penalty is imposed on the terminal time, then this creates a "free pass" structure in which the value of the objective can be improved without incurring a cost. In such a situation, optimal solutions need not exist. Intuitively, the controls switch to $u=0$ immediately as the separatrix is crossed and then take an increasingly longer time as they pass near the saddle point with the infimum arising in the limit $T \rightarrow \infty$. From a practical point of view it anyhow is unacceptable for the system to move along the boundary between benign and malignant behaviors. In view of imprecise and mathematically unmodeled dynamics and other random perturbations, the addition of this term provides desired robustness and stability properties for the underlying real system. Thus it makes perfect sense, both mathematically and practically, to include a penalty term on the final time in the objective. This creates a well-posed mathematical problem for which the existence of solutions follows from standard theory.

\subsection{Structure of optimal protocols}

As for the models considered above, an analysis of the conditions of the maximum principle once more leads to bang and singular controls as candidates for optimality. The computation of singular controls and corresponding singular arcs gives the following result:

Proposition 4.2. [21] For the optimal control problem [C-TI], singular arcs are contained in the 1dimensional set

$$
\mathcal{S}=\left\{(x, y) \in \mathbb{M}: P(x, y)=p_{2}(x) y^{2}+p_{1}(x) y+p_{0}=0\right\}
$$

where

$$
\begin{aligned}
p_{0} & =C \alpha \mu_{C}=\text { const } \\
p_{1}(x) & =\left[-C \mu_{C} \log \left(\frac{x}{x_{\infty}}\right)+D \kappa\right] \mu_{I}\left(x-2 \beta x^{2}\right)+\mu_{C} C\left(\mu_{I}\left(x-\beta x^{2}\right)-\delta\right), \\
p_{2}(x) & =-C \gamma \mu_{I}\left(x-2 \beta x^{2}\right) .
\end{aligned}
$$

The singular control that keeps the set $\mathcal{S}$ invariant is given in feedback form as

$$
u_{\text {sing }}(t)=-\frac{\varphi_{1}\left(z_{*}(t)\right)}{\theta_{1}\left(z_{*}(t)\right)}
$$

with the coefficients $\varphi_{1}$ and $\theta_{1}$ defined by expressing the Lie brackets $[f,[f, g]]$, respectively $[g,[f, g]]$, as a linear combination of $g$ and $[f, g]$ in the form

$$
[f,[f, g]](z)=\varphi_{1}(z) g(z)+\varphi_{2}(z)[f, g](z)
$$

and

$$
[g,[f, g]](z)=\theta_{1}(z) g(z)+\theta_{2}(z)[f, g](z) .
$$

The strengthened Legendre-Clebsch condition for optimality of a singular extremal is satisfied for $x<\frac{1}{4 \beta}$ and $\frac{1}{2 \beta}<x$, and it is violated for $\frac{1}{4 \beta}<x<\frac{1}{2 \beta}$.

Again optimal controls need to be synthesized from bang and singular arcs and the potential presence of optimal singular arcs makes numerical computations challenging. Below we give an example of a numerically computed optimal control [21] for which the classical $\varepsilon$-algorithm approach was used with 
a quadratic penalty term $\varepsilon \int_{0}^{T} u^{2}(t) d t$ added to the objective and then the optimal controls for the underlying problem were recovered in the limit as $\varepsilon \rightarrow 0$. For the actual computations, carried out by M. Naghnaeian, we used GPOPS (General Pseudo-spectral OPtimal Control $S$ oftware), an open-source MATLAB optimal control software that implements the Gauss hp-adaptive pseudo-spectral methods (http://www.gpops.org/, [40]). In our simulations, following a brief initial interval when chemotherapy is given at full dose rate, it is a singular arc that typically moves the state of the system from the malignant into the benign region. The analytic formulas derived above allowed us to verify that the numerically found solutions indeed were accurate in the sense that the corresponding controlled trajectories followed the singular curve $\mathcal{S}$ along singular controls. In some in a certain sense extreme cases that arise when the coefficients $C$ and $S$ in the objective (4.6) skew the importance of the side-effects versus the terminal time, optimal controls give full-dose chemotherapy over the full interval $[0, T]$. But aside from these special scenarios, optimal solutions always contained a time interval when the control was singular.

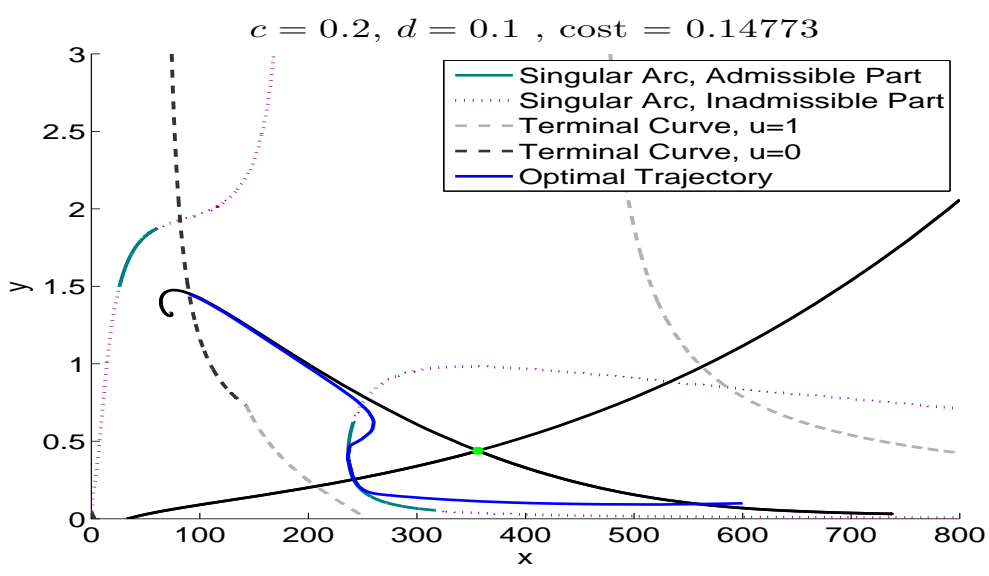

FiguRE 6. Example of a numerically computed optimal controlled trajectory.

Figure 6 shows the graph of a numerically computed optimal trajectory (blue curve) from the initial condition $\left(x_{0}, y_{0}\right)=(600,0.1)$. The tumor volume $x_{0}$ is given as a multiple of some reference value and represents a tumor cell count that is 600 times higher than some chosen base value (say $10^{6}$ cells); $y_{0}$ is a dimensionless, order-of-magnitude quantity that represents a depletion of the immuno-competent cell densities to $10 \%$ of a nominal value. This initial condition lies well within the malignant region. In the objective, we used the coefficients $A$ and $B$ determined by the stable eigenvector of the saddle for the data given in Table $1, A=0.00192$ and $B=1$. The penalty terms were chosen as $C=0.2$ and $D=0.1$ and the numerical value chosen for $\kappa$ is $\kappa=1$. For these coefficients, initially the control is given by $u \equiv 1$ over some interval $\left[0, t_{1}\right]$ at which time the control becomes singular. The optimal controlled trajectory then follows the singular arc from the malignant into the benign region across the separatrix. In the benign region, at a certain time $\tau$ the control switches to $u \equiv 0$ and then follows the uncontrolled trajectory towards the benign equilibrium point. If the penalty on the chemotherapeutic agent is not too high, then optimal controls actually still switch for one more time to a full dose chemotherapy segment at the end of treatment, possibly after a prolonged period of rest. This structure can also be used to define a three-dimensional minimization problem for the switching times whose numerical solution defines the optimal control.

\subsection{Comments and Interpretation}

There are some interesting theoretical insights about optimal therapies in the presence of tumor immune interactions that can be drawn from these numerical computations. By including a penalty term on 
the final time $T$, we have given a well-posed formulation for which optimal controls exist. If too much prominence is given to this penalty, optimal controls will simply be constant maximum dose therapies, i.e., if time is of the essence, give as much as you can, as soon as you can. However, if the time horizon is longer and the coefficient at the terminal time is lowered, optimal responses are concatenations that start with a full dose therapy session, but then are followed by a segment when the dose rates are lowered and given according to a singular control. As some of the examples show, during such a period, it is even possible that the tumor volume $x$ increases again. However, the immunocompetent density $y$ increases and leads to an overall better state that lies in the benign region. Thus, optimal controls no longer aim at eradicating the tumor, but rather are content to move the state of the system into a region where the innate beneficial actions of the immune system are able to control the cancer. These strategies correspond to protocols that initially apply a burst of chemotherapy to reduce the tumor volume and then sustain a smaller volume with reduced dosages. In the medical literature, such protocols have been considered under the terminology of "chemo-switch" strategies. The additional, and usually very short burst of full dose chemotherapy that marks the end of some of these therapies also is quite interesting. While this may appear a bit odd at first, there indeed exist practical chemotherapy protocols that follow such a pattern.

With the prominent role played by the singular arc, these solutions for model [C-TI] contrast with the optimal bang-bang controls for cell-cycle specific models for cancer chemotherapy considered earlier when no tumor-immune system interactions were taken into account. It is the mitigating influence of the immune system which for smaller tumor volumes leads to the abandonment of the strict bang-bang scheme that is seen in the cell-cycle specific models. Intuitively, if the system is in a condition where it is able to control the cancer itself, why administer chemotherapy that in fact may destroy this innate ability of the organism? Thus, despite the model's simplicity, its solutions address the important practical question how to schedule therapies over time and lead to some qualitative structures that can be considered an initial step towards designing treatment protocols for more complex models.

\section{Conclusion}

The question how chemotherapeutic agents for cancer should be scheduled in time has not always a satisfactory answer. Clearly, there are situations when the classical MTD paradigm is appropriate and effective. But there are also situations when it fails. The mathematical models considered above, as simplified as they are, and their analysis by means of optimal control, gives information that helps to shed some light onto what kind of protocols may be preferred in what situations. The qualitative structure of optimal controls changes from solutions that favor maximum dose rate therapies with restperiods in case of homogeneous, chemotherapeutically sensitive populations of cancer cells, to possible lower dose rate administrations for heterogeneous tumor populations to optimal controls that contain singular arcs with significantly reduced dose rates as tumor immune system interactions are taken into consideration. Interestingly, solutions of the type seen here for the model with tumor immune interactions have also been investigated in medical research as "chemo-switch" type protocols [36]. A medical argument can be made why in some situations lower dose rates, as they are inherently arise with optimal singular controls, may be preferred: by administering drugs in a continuous low-dose way, it may be possible to avoid the limiting toxic side effects of high dose therapy and, ideally, the overall effect of therapy may be improved because of the greatly extended time horizon over which drugs can be given $[14,16]$. There exist various modi operandi that have been proposed in the medical literature to explain the effectiveness that this kind of therapy has shown in childhood cancers (see, for example the review articles $[1,35]$ and the various medical references cited therein) and this has led to the pursuit of a so-called metronomic scheduling for chemotherapy in medical trials (e.g., see $[2,15]$ ). There is mounting medical evidence that for some cancers more need not necessarily be better, but that a properly calibrated, so-called biologically optimal or minimum biologically effective dose, may be preferred. In the year 2000, several papers addressed this aspect $[5,15,19]$ introducing the concept which is now named metronomic chemotherapy. It was defined as the frequent administration of chemotherapeutic drugs at relatively low, nontoxic doses without prolonged 
drug breaks. In addition to the expected cytotoxic effect, metronomic treatment exhibits reduced toxicity to healthy cells and surprisingly also impacts some aspects of the tumor microenvironment positively. Specifically, some antiangiogenic effects have been observed as well as a boost to the immune system [35]. One of the reasons supporting MTD chemotherapy, the argument that chemotherapy at lower doses prompts the development of drug resistance, has also been challenged by experimental data coming from metronomic treatment. It has been observed that under properly calibrated lower dose chemotherapy the process of re-sensitization took place, i.e., some resistant cell populations became again sensitive to the drug.

In view of these benefits, it becomes natural that the search for a biologically optimal dose is worthwhile to pursue and mathematical modeling has a role here. More specifically, since it is not known how metronomic chemotherapy should be administered, i.e., what exactly the biologically optimal dose is (besides rough estimates that it should lie between $10 \%$ and $80 \%$ of MTD), methods from optimal control can shed some light on this issue. Singular controls, along with the reduced dose rates with which they normally arise and which some time ago were dismissed as an oddity when it came to the scheduling of agents, here offer interesting insights into what might be a biologically optimal dose.

Acknowledgements. This material is based upon work supported by the National Science Foundation under collaborative research Grants Nos. DMS 1311729/1311733. Any opinions, findings, and conclusions or recommendations expressed in this material are those of the author(s) and do not necessarily reflect the views of the National Science Foundation.

\section{References}

[1] N. André, L. Padovani, E. Pasquier. Metronomic scheduling of anticancer treatment: the next generation of multitarget therapy? Fut. Oncology, 7(3) (2011), 385-394.

[2] N. Andre, S. Abed, D. Orbach, C. Armari Alla, L. Padovani, E. Pasquier, J.C. Gentet, A. Verschuur. Pilot study of a pediatric metronomic 4-drug regimen. Oncotarget, 2 (2011), 960-965.

[3] G. Bocci, K. Nicolaou, R.S. Kerbel. Protracted low-dose effects on human endothelial cell proliferation and survival in vitro reveal a selective antiangiogenic window for various chemotherapeutic drugs. Cancer Res., 62 (2002), 6938-6943.

[4] B. Bonnard, M. Chyba. Singular Trajectories and their Role in Control Theory. Mathématiques \& Applications, Vol. 40. Springer Verlag, Paris, 2003.

[5] T. Browder, C.E. Butterfield, B.M. Kräling, B. Shi, B. Marshall, M.S. O'Reilly, J. Folkman. Antiangiogenic scheduling of chemotherapy improves efficacy against experimental drug-resistant cancer. Cancer Res., 60 (2000), $1878-1886$.

[6] M. Eisen. Mathematical Models in Cell Biology and Cancer Chemotherapy. Lecture Notes in Biomathematics, Vol. 30. Springer Verlag, 1979.

[7] A. Friedman. Cancer as Multifaceted Disease. Math. Model. Nat. Phenom., 7 (2012), 1-26.

[8] R.A. Gatenby, A.S. Silva, R.J. Gillies, B.R. Frieden. Adaptive therapy. Cancer Research, 69 (2009), $4894-4903$.

[9] J.H. Goldie. Drug resistance in cancer: a perspective. Cancer and Metastasis Review, 20 (2001), 63-68.

[10] J.H. Goldie, A. Coldman. Drug Resistance in Cancer. Cambridge University Press, 1998.

[11] J. Greene, O. Lavi, M. Gottesman, D. Levy. The impact of cell density and mutations in a model of multidrug resistance in solid tumors. Bull. Math. Biol., 74 (2014), 627-653.

[12] J. Guckenheimer, P. Holmes. Nonlinear Oscillations, Dynamical Systems, and Bifurcations of Vector Fields. Springer Verlag, New York, 1983.

[13] P. Hahnfeldt, L. Hlatky. Cell resensitization during protracted dosing of heterogeneous cell populations. Rad. Res., 150 (1998), 681-687.

[14] P. Hahnfeldt, J. Folkman, L. Hlatky. Minimizing long-term burden: the logic for metronomic chemotherapy dosing and its angiogenic basis. J. of Theo. Biol., 220 (2003), 545-554.

[15] D. Hanahan, G. Bergers, E. Bergsland. Less is more, regularly: metronomic dosing of cytotoxic drugs can target tumor angiogenesis in mice. J. Clin. Invest., 105(8) (2000), 1045-1047.

[16] B. Kamen, E. Rubin, J. Aisner, E. Glatstein. High-time chemotherapy or high time for low dose? J. Clinical Oncology, 18(16) (2000), 2935-2937.

[17] T.J. Kindt, B.A. Osborne, R.A. Goldsby. Kuby Immunology. W.H. Freeman, 2006.

[18] D. Kirschner, J.C. Panetta. Modeling immunotherapy of the tumor-immune interaction. J. of Math. Biol., 37 (1998), $235-252$.

[19] G. Klement, S. Baruchel, J. Rak, S. Man, K. Clark, D.J. Hicklin, P. Bohlen, R.S. Kerbel. Continuous low-dose therapy with vinblastine and VEGF receptor-2 antibody induces sustained tumor regression without overt toxicity. J. Clin. Invest., 105(8) (2000), R15-R24. 
[20] V.A. Kuznetsov, I.A. Makalkin, M.A. Taylor, A.S. Perelson. Nonlinear dynamics of immunogenic tumors: parameter estimation and global bifurcation analysis. Bull. Math. Biol., 56 (1994), 295-321.

[27] U. Ledzewicz, K. Bratton, H. Schättler. A 3-compartment model for chemotherapy of heterogeneous tumor populations. Acta Mat. Appl., (2014), to appear.

[21] U. Ledzewicz, M. Naghnaeian, H. Schättler. Optimal response to chemotherapy for a mathematical model of tumorimmune dynamics. J. of Math. Biol., 64 (2012), 557-577.

[22] U. Ledzewicz, O. Olumoye, H. Schättler. On optimal chemotherapy with a stongly targeted agent for a model of tumorimmune system interactions with generalized logistic growth. Math. Biosci. Engr. (MBE), 10(3) (2012), 787-802.

[23] U. Ledzewicz, H. Schättler. Optimal bang-bang controls for a 2-compartment model in cancer chemotherapy. J. of Optim. Th. Appl. (JOTA), 114 (2002), 609-637.

[24] U. Ledzewicz, H. Schättler. Analysis of a cell-cycle specific model for cancer chemotherapy. J. of Biol. Syst., 10 (2002), 183-206.

[25] U. Ledzewicz, H. Schättler. Drug resistance in cancer chemotherapy as an optimal control problem. Discr. Cont. Dyn. Syst., Ser. B, 6 (2006), 129-150.

[26] U. Ledzewicz, H. Schättler, M. Reisi Gahrooi, S. Mahmoudian Dehkordi. On the MTD paradigm and optimal control for combination cancer chemotherapy. Math. Biosci. Engr. (MBE), 10 (2013), 803-819.

[28] R. Martin, K.L. Teo. Optimal Control of Drug Administration in Cancer Chemotherapy. World Scientific Publishers, 1994.

[29] A. Matzavinos, M. Chaplain, V.A. Kuznetsov. Mathematical modelling of the spatio-temporal response of cytotoxic T-lymphocytes to a solid tumour. Math. Med. Biol., 21 (2004), 1-34.

[30] A. d'Onofrio. A general framework for modelling tumor-immune system competition and immunotherapy: Mathematical analysis and biomedial inferences. Physica D, 208 (2005), 202-235.

[31] A. d'Onofrio. Tumor evasion from immune control: strategies of a MISS to become a MASS. Chaos, Solitons and Fractals, 31 (2007), 261-268.

[32] A. d'Onofrio. Metamodeling tumor-immune system interaction, tumor evasion and immunotherapy. Math. Comp. Modelling, 47 (2008), 614-637.

[33] D. Pardoll. Does the immune system see tumors as foreign or self? Ann. Rev. of Immun., 21 (2003), 807-839.

[34] E. Pasquier, U. Ledzewicz. Perspective on "More Is Not Necessarily Better": Metronomic Chemotherapy. SMB Newsletter, 26(3) 2013, 9-10.

[35] E. Pasquier, M. Kavallaris, N. André. Metronomic chemotherapy: new rationale for new directions. Nat. Rev.| Clin. Onc., 7 (2010), 455-465.

[36] K. Pietras, D. Hanahan. A multi-targeted, metronomic and maximum tolerated dose "chemo-switch" regimen is antiangiogenic, producing objective responses and survival benefit in a mouse model of cancer. J. Clin. Onc., 23(5) (2005), 939-952.

[37] L.G. de Pillis, A. Radunskaya. A mathematical tumor model with immune resistance and drug therapy: an optimal control approach. J. Theo. Med., 3 (2001), 79-100.

[38] L.G. de Pillis, A. Radunskaya, C.L. Wiseman. A validated mathematical model of cell-mediated immune response to tumor growth. Cancer Res., 65 (2005), 7950-7958.

[39] L.S. Pontryagin, V.G. Boltyanskii, R.V. Gamkrelidze, E.F. Mishchenko. The Mathematical Theory of Optimal Processes, MacMillan, New York, 1964.

[40] A.V. Rao, D.A. Benson, G.T. Huntington, C. Francolin, C.L. Darby, M.A. Patterson. User's Manual for GPOPS: A MATLAB Package for Dynamic Optimization Using the Gauss Pseudospectral Method. University of Florida Report, 2008.

[41] H. Schättler, U. Ledzewicz. Geometric Optimal Control. Springer, New York, 2012.

[42] H. Schättler, U. Ledzewicz, S. Mahmoudian Dehkordi, M. Reisi Gahrooi. A geometric analysis of bang-bang extremals in optimal control problems for combination cancer chemotherapy. Proc. of the 51st IEEE Conference on Decision and Control, Maui, Hawaii, December 2012, 7691-7696.

[43] N.V. Stepanova. Course of the immune reaction during the development of a malignant tumour. Biophys., 24 (1980), 917-923.

[44] G.W. Swan. Role of optimal control in cancer chemotherapy. Math. Biosci., 101 (1990), $237-284$.

[45] J.B. Swann, M.J. Smyth. Immune surveillance of tumors. J. Clin. Invest., 117 (2007), 1137-1146.

[46] A. Swierniak. Optimal treatment protocols in leukemia - modelling the proliferation cycle. Proc. 12th IMACS World Congress, Paris, 4 (1988), 170-172.

[47] A. Swierniak. Cell cycle as an object of control. J. of Biol. Syst., 3 (1995), 41-54.

[48] A. Swierniak, U. Ledzewicz, H. Schättler. Optimal control for a class of compartmental models in cancer chemotherapy. Int. J. Appl. Math. Comp. Sci., 13 (2003), 357-368.

[49] A. Swierniak, A. Polanski, M. Kimmel. Optimal control problems arising in cell-cycle-specific cancer chemotherapy. Cell Prolif., 29 (1996), 117-139.

[50] A. Swierniak, J. Smieja. Cancer chemotherapy optimization under evolving drug resistance. Nonlin. Anal., 47 (2000), 375-386.

[51] H.P. de Vladar, J.A. González. Dynamic response of cancer under the influence of immunological activity and therapy. J. of Theo. Biol., 227 (2004), 335-348.

[52] S.D. Weitman, E. Glatstein, B.A. Kamen. Back to the basics: the importance of concentration $\times$ time in oncology. J. of Clin. Onc., 11 (1993), 820-821. 